\title{
Nonlinear flow in low permeability reservoirs: Modelling and experimental verification
}

\author{
Fuquan Song ${ }^{1 \oplus *}$, Liwen Bo ${ }^{1}$, Shiming Zhang ${ }^{2}$, Yeheng Sun $^{2}$ \\ ${ }^{1}$ College of Petrochemical and Energy Engineering, Zhejiang Ocean University, Zhoushan 316022, P. R. China \\ ${ }^{2}$ Shengli Oilfield Company of China Petroleum \& Chemical Co, Ltd, Exploration and Development Research Institute, \\ Dongying 257015, P. R. China
}

(Received October 28, 2018; revised November 23, 2018; accepted November 25, 2018; available online December 1, 2018)

\section{Citation:}

Song, F., Bo, L., Zhang, S., Sun, Y. Nonlinear flow in low permeability reservoirs: Modelling and experimental verification. Advances in Geo-Energy Research, 2019, 3(1): 76-81, doi: 10.26804/ager.2019.01.06.

Corresponding author: *E-mail: songfuquan@zjou.edu.cn

\section{Keywords:}

Low permeability reservoirs non-Darcy seepage threshold pressure gradient boundary adhesion layer slip length

\begin{abstract}
:
The low-permeability reservoirs are of heavy heterogeneity, low permeability, fine oil-water passages, strong resistance during flow, and the significant interaction between solid and liquid interfaces causes the flow of fluid in reservoir deviating from the Darcy's law. There is no agreement on the interaction between the various factors in seepage process and the influence of seepage law. The boundary layer exists when liquid flow in micro-tubes and nano-tubes, and the boundary layer decreases with the increase of driving force, and the maximum value of boundary layer ratio is equal to 1 . Based on the capillary boundal model and the boundary layer theory, a new exponential seepage model for low permeability reservoirs was proposed. Some experiments of water flow with different pressure gradient were carried out in low permeability cores with permeability of 4 to 8 milidarcy in natural rock cores from an oilfield in China, and the nonlinear model is of good agreement with the single-phase water flooding experiments of these cores. The results demonstrate that the physical meaning of each parameter of the new model is clear and it can be applied to describe the nonlinear characteristics of low permeability reservoirs. The large driving force can overcome disadvantages in the developments of low permeability reservoirs.
\end{abstract}

\section{Introduction}

In recent years, with the continuous consumption of oil and gas resources, the medium and high permeability reservoir resources have been inevitably reduced, and the low permeability reservoirs are gradually put into exploitation and utilization (Wang et al., 2011). The flow law no longer conforms to Darcy's law when it comes to low permeability due to the poor permeability, the complex pore structure and the poor connectivity, which is characterized by nonlinear seepage flow, and the presence of threshold pressure gradient in the flow process (Feng et al., 2010). That is to say, the boundary fluid on the surface of rock particles needs some external force to flow under the action of solid-liquid interface (Dong et al., 2013).

With the increasing reserves of low permeability reservoirs (Wei et al., 2011), a large number of scholars have studied the nonlinear seepage model above, which is known as gradient model, and widely used (Li et al., 2014; Song et al., 2018). The point of $G_{s}$ shown in Fig. 1 is defined as the pseudo-starting

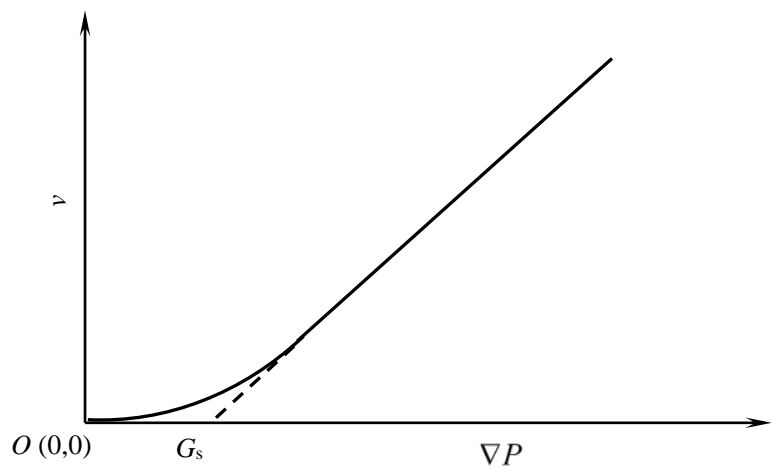

Fig. 1. Seepage curve of fluid through porous media in low permeability reservoir (Peng et al., 2012).

pressure gradient. It's an intersection with the pressure axis when the straight line of the flow-pressure gradient curve is extended forward (Huang, 1997; Zao et al., 2012). The model neglects the curve section under small pressure gradient, and 


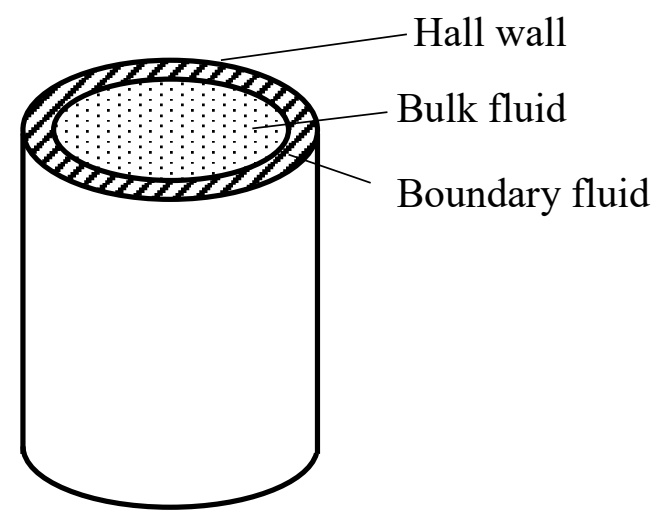

Fig. 2. The schematic diagram of seepage fluid occurrence (Liu et al., 2011).

the seepage model is (Deng et al., 2001):

$$
\vec{v}= \begin{cases}0, & |\nabla p| \leq G_{s} \\ -\frac{K}{\mu}\left(1-\frac{G_{s}}{|\nabla p|}\right) \nabla p, & |\nabla p|>G_{s}\end{cases}
$$

where $v$ is velocity of fluid flow, $K$ is permeability of porous media, $\mu$ is viscosity of fluid, $G_{s}$ is threshold pressure gradient, $p$ is pressure of fluid.

Yao et al. (2000) analysed core experimental data in the dimensionless analysis method, then he obtained a piecewise seepage model:

$$
\begin{array}{ll}
v=0 & \text { for super-low speed zone, } \\
v=c\left(\frac{\Delta p}{L}\right)^{\frac{1}{2-n}} & \text { for low speed transition zone, } \\
v=\frac{K}{\mu} \frac{\Delta p}{L} & \text { for Darcy flow zone. }
\end{array}
$$

where $L$ is length of cores, $c$ and $n$ are constant numbers.

This model divides the seepage into three sections, which reflects the flow state of the liquid in the reservoir. But it's of less convenience in practical appliance because of the complexity and the heavy calculation burden.

Taking the lithology and fluid characteristics of low permeability reservoirs into account, Yang Zhengming (Yang et al., 2010) came up with a nonlinear seepage model of discriminant type:

$$
v=\frac{K}{\mu} \nabla p\left(1-\frac{G_{s}}{\nabla p}\right)
$$

when $K$ is a constant and $G_{s}$ is zero, the formula conforms to Darcy's law; when $K$ is a constant and $\delta$ is a constant, the formula becomes a non-Darcy flow model with considering the starting pressure gradient; when $K$ is a variable, $G_{s}$ is zero, the formula is suitable for ultra-low permeability reservoirs.

Considering the nonlinear parameter of seepage, Jiang et al. (2011) gave a complete function model:

$$
\vec{v}=\frac{K}{\mu}\left(1-\frac{4 c_{1}}{|\nabla p|}+\frac{6 c_{1}^{2}}{|\nabla p|\left(|\nabla p|-c_{1}\right)}\right) \nabla p
$$

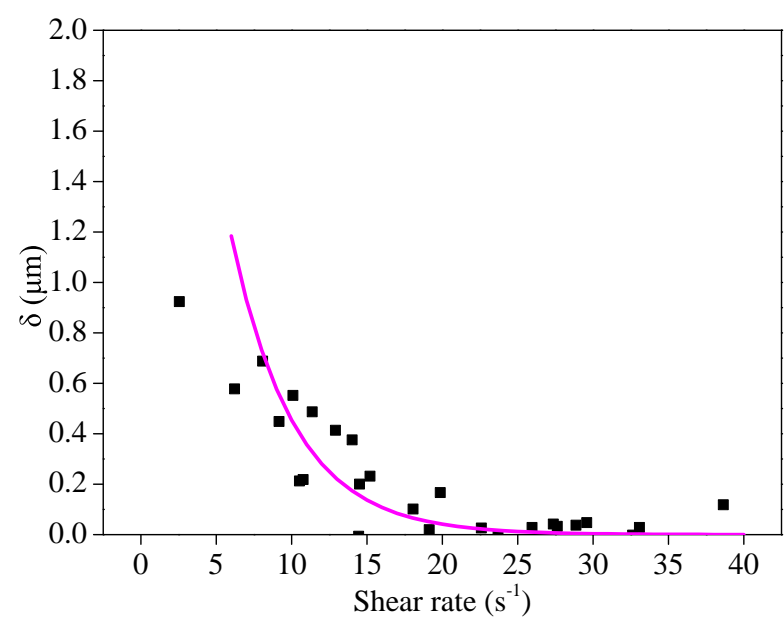

Fig. 3. The relationship between the thickness of the boundary layer and the shear rate in the microcircular tube ( $r=5 \mathrm{~mm}$ ) (Huang et al., 2013).

where $c_{1}$ is the nonlinear parameter of seepage.

Through abundant experimental studies and mathematical derivations, we found that microscopic parameters such as reservoir physical properties, fluid properties and solid-liquid interfaces interaction have different degrees of influence on nonlinear seepage in low permeability reservoirs, and they have certain guidance for the development of low permeability reservoirs (Zhao et al., 2014; Liu et al., 2015; Li et al., 2016). Based on the previous research results and the boundary layer theory, a new nonlinear seepage model for low permeability reservoirs is proposed. And the model has been verified by the single-phase water flooding experiment in natural rock cores of a domestic oilfield.

\section{Nonlinear seepage model in low permeability reservoirs}

Huang et al. (2013) believed that in porous media, the liquid molecules are orderly distributed on the solid-liquid interface due to the intermolecular forces at the solid-liquid interface. On this basis, the concept of seepage fluid and its physical model in porous media are raised, as shown in Fig. 2. He pointed out that the seepage fluid includes two parts: The bulk fluid and the boundary fluid, and the boundary fluid is immobile because of the interface phenomenon.

In a wettable microcircular tube, the fluid called a boundary adhesion layer is adsorbed on a solid surface and does not participate in the flow. The thickness of the boundary fluid is affected by the fluid properties, the pore structure of the porous medium and the displacement pressure, and it varies with the displacement pressure. It indicates in Fig. 3 that the bulk fluid participates in the fluid flow when under the displacement pressure (Tian et al., 2016). The results of microtubule experiments figure that (Song et al., 2016): In the same microtubule, the larger the pressure gradient, the thinner the boundary viscous layer, and the thickness of the boundary layer presents an exponential relationship with the displacement pressure gradient.

Fig. 3 illustrates that when the driving force approaches 0 , 


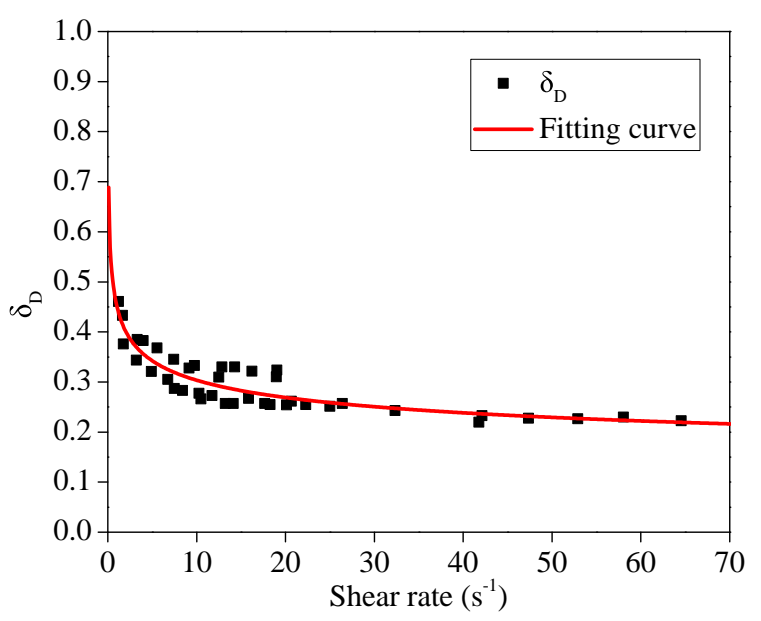

Fig. 4. The relationship between the thickness of the boundary layer and the shear rate in the nano-tube $(r=26 \mathrm{~nm})$.

only boundary fluid exists in the seepage fluid ( $\mathrm{Xu}$ et al., 2007; Zhang et al., 2008; Li et al., 2011), so the radius of the microtube is the thickness of the boundary adhesion layer in the microcircular tube. Thus the thickness model of the boundary adhesion layer turns out as follows:

$$
\delta=\delta_{D} r_{0} e^{-c|\nabla p|}
$$

where $\delta$ is the thickness of the boundary adhesion layer, the unit is $\mathrm{m} ; r_{0}$ is the radius of the micro-circular tube, the unit is $\mathrm{m}$; $c$ is the boundary adhesion layer parameter, the unit is $\mathrm{m} \cdot \mathrm{MPa}^{-1} ; \delta_{0}$ is the maximum boundary layer thickness and $r_{0}$ is radius of microtubes; $\delta_{D}=\delta_{0} / r_{0}$ is the ratio of the maximum boundary layer thickness.

According to the stress-strain relationship of Newtonian fluid:

$$
\tau=\mu \gamma
$$

where $\tau$ is the stress of the fluid, $\mathrm{Pa} ; \gamma$ is the strain of the fluid, $\mathrm{s}^{-1}$.

By deriving the Eq. (6), we can derive the flow formula in the microcircular tube.

$$
q=\int_{0}^{r_{0}-\delta} v(r) \cdot 2 \pi r d r=\frac{\pi\left(1-\delta_{D} e^{-c|\nabla p|}\right)^{4} r_{0}^{4}}{8 \mu}|\nabla p|
$$

We have carried out an experiment of oil flow in nanotube, the outcome of the variation of the boundary layer ratio with the shear rate is shown in Fig. 4. It turns out that: When the shear rate is small, the proportion of the boundary layer decreases rapidly with the increase of the shear rate, when the shear rate is large, the proportion of the boundary layer slowly decreases as the shear rate increases, and gradually tends to be constant. Through the power function fitting, it can be found that when the shear rate is close to 0 , the fitting value of the boundary layer ratio is close to 1 , which means $\delta_{D}=1$. Then Eq. (7) can be simplified as:

$$
q=\frac{\pi\left(1-e^{-c|\nabla p|}\right)^{4} r_{0}^{4}}{8 \mu}|\nabla p|
$$

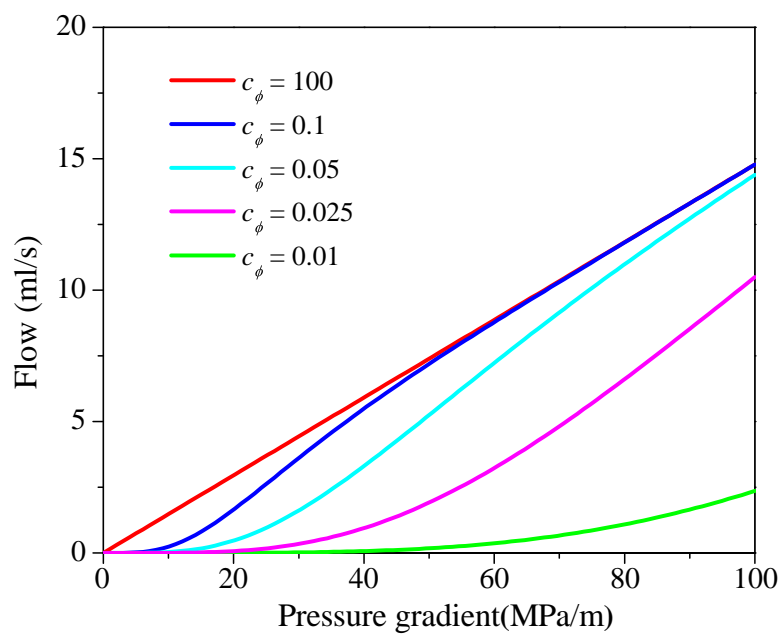

Fig. 5. Relationship between pressure gradient and flow rate in lowpermeability core.

For porous media, through analyzing the corresponding seepage formula with the capillary flow model, the seepage formula in low permeability porous media can be obtained as:

$$
Q=\sum_{i=1}^{N} n_{i} q\left(r_{i}\right)=\sum_{i=1}^{N} n_{i} \frac{\pi\left(1-e^{-c_{i}|\nabla p|}\right)^{4} r_{i}^{4}}{8 \mu}|\nabla p|
$$

Then, the seepage formula is as follows:

$$
\vec{v}=\frac{K_{0}\left(1-e^{-c_{\phi}|\nabla p|}\right)^{4}}{\mu} \nabla p
$$

where $\phi=\frac{\sum_{i=1}^{N} n_{i} \pi r_{i}^{2}}{A}, \quad K_{0}=\frac{\phi}{8} \frac{\sum_{i=1}^{N} n_{i} r_{i}^{4}}{\sum_{i=1}^{N} n_{i} \pi r_{i}^{2}}, \quad c_{\phi}$ is the average boundary adhesion layer parameters.

Assuming that the simulated formation water flows in a core that $L=60 \mathrm{~mm}, d=25 \mathrm{~mm}, k=2.83 \mathrm{mD}$. The flow rate of the simulated formation water under the influence of different $c_{\phi}$ values can be obtained by the formula, as shown in Fig. 5. There are three cases of $c_{\phi}$ values: When $c_{\phi}$ is large, the simulated formation water flow characteristics are close to Darcy flow; when $c_{\phi}$ is small, under small pressure gradient, the flow rate decreases, the characteristics do not conform to Darcy's law; when $c_{\phi}$ tends to infinite, Eq. (10) is Darcy's law.

\section{Experimental verification of nonlinear seepage model in low permeability reservoirs}

For a further validation, we adopt the steady-state method to conduct a single-phase water flooding experiment on the six natural cores of low-permeability reservoirs in an oilfield, then analyze the nonlinear seepage. The specific experimental contents are as follows.

\subsection{Experimental materials and equipment}

The experimental water is simulated formation water, the salinity is $10,587 \mathrm{mg} / \mathrm{L}$. The basic parameters of the core used 
Table 1. The basic parameters of the cores.

\begin{tabular}{lllll}
\hline Cores & Length/cm & Diameter/cm & Permeability $/ \mathrm{mD}$ & Porosity $/ \%$ \\
\hline Core-1 & 5.211 & 2.507 & 8.33 & 9.03 \\
Core-2 & 5.605 & 2.509 & 7.24 & 9.43 \\
Core-3 & 7.288 & 2.507 & 6.30 & 13.00 \\
Core-4 & 6.885 & 2.510 & 5.87 & 17.81 \\
Core-5 & 6.546 & 2.507 & 5.40 & 13.97 \\
Core-6 & 5.285 & 2.490 & 4.20 & 9.08 \\
\hline
\end{tabular}

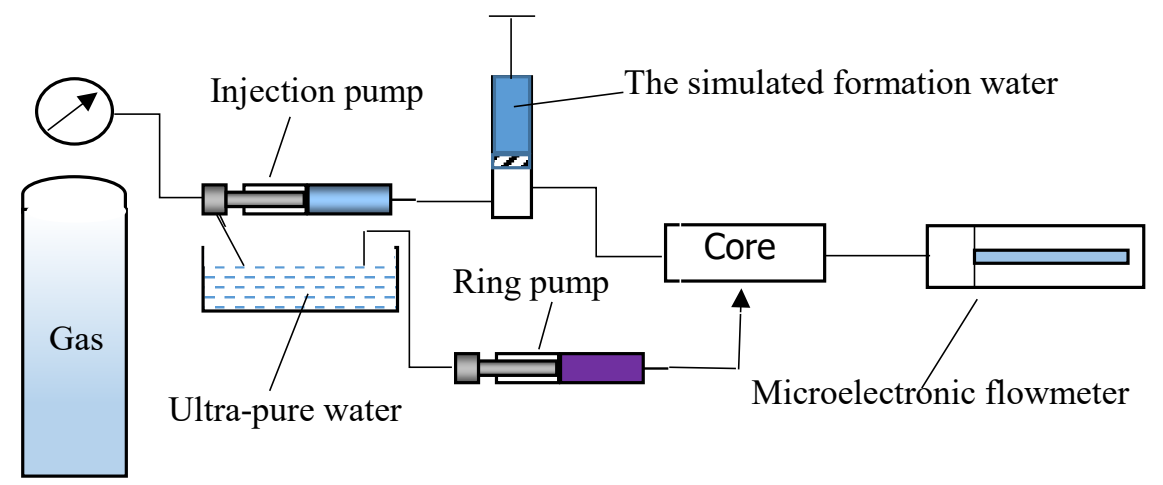

Fig. 6. Flow chart of single-phase water flooding experiment.

are shown in Table 1.

The experimental equipment includes: $\mathrm{BH}-3$ the experimental device of vacuum and forcing (oil or water) for cores; QC-I the device of testing recovery by gas flooding; double cylinder tracking pump in constant speed and constant pressure (injection pump); single cylinder tracking pump in constant speed and constant pressure (ring pump); microelectronic flowmeter.

\subsection{Experimental steps}

The experimental process is displayed in Fig. 6. The experimental steps are as below: (1) Through BH-3 the experimental device of vacuum and forcing (oil or water) for cores, saturate the simulated formation water for $48 \mathrm{~h}$ in setting the pressure for $30 \mathrm{MPa}$ after vacuumizing the cores for $5 \mathrm{~h}$; (2) Connect experimental device pipelines and put the cores into core holder; (3) Open the nitrogen gas and injection pump, and injects the simulated formation water at a constant pressure from 10-1 $\mathrm{MPa}$ at the entrance. The ring pump is $5 \mathrm{MPa}$ higher than injection pressure; (4) The flow rate at exit is measured by microelectronic flow-meter, and the flow rate value under each displacement pressure is recorded after the flow rate is stabilized; (5) The core is replaced, and steps (1)(4) are repeated.

\subsection{Experimental results and analysis}

According to the above experimental steps, we can obtain the relationship between the flow and pressure gradient of the six cores.

It's obvious that the phenomenon of nonlinear seepage of the core is evident as can be seen from the figure, and the new seepage model of the low permeability reservoir can fit the experimental data well with high fitting coefficient.

\section{Conclusions}

Based on the previous research results and the boundary layer theory, a new and single nonlinear seepage model for low permeability reservoirs is proposed. And the model is verified by the single-phase water flooding experiment in natural rock cores of a domestic oilfield. And it comes to the conclusions:

1) The boundary layer of liquid flow in nano- and microtubes changes according to driving force, and the boundary layer height ranges from 1 to tube diameter with the increase of the driving force. And a new nonlinear seepage model for low permeability reservoirs is established on the basis of the boundary layer theory.

2) Since the experiments of the water flow in permeability cores have been accomplished, the nonlinear model is proved to be credible to depict the nonlinear characteristics of low permeability reservoirs.

\section{Acknowledgments}

The first author acknowledges the major program of the National Natural Science Foundation of China (No. 2017ZX05072005) and the National Natural Science Foundation of China (No. 11472246) for the financial support to 


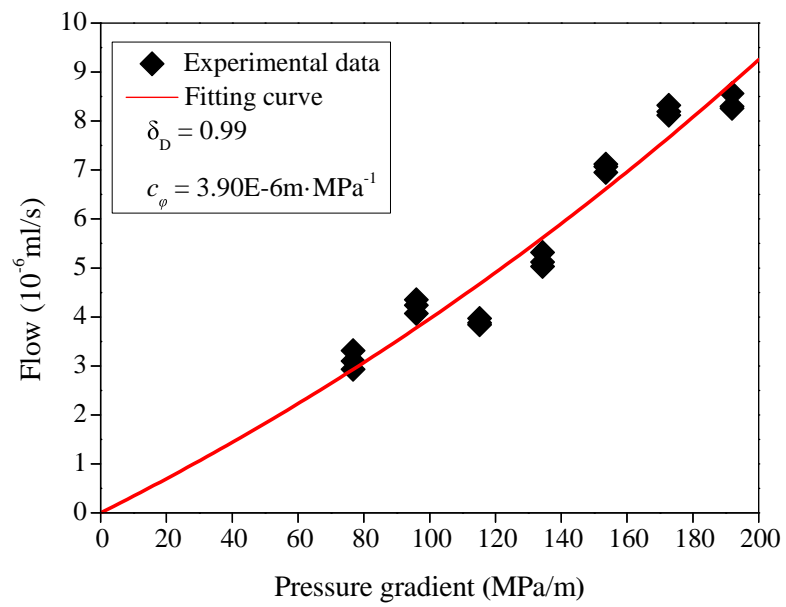

(a)

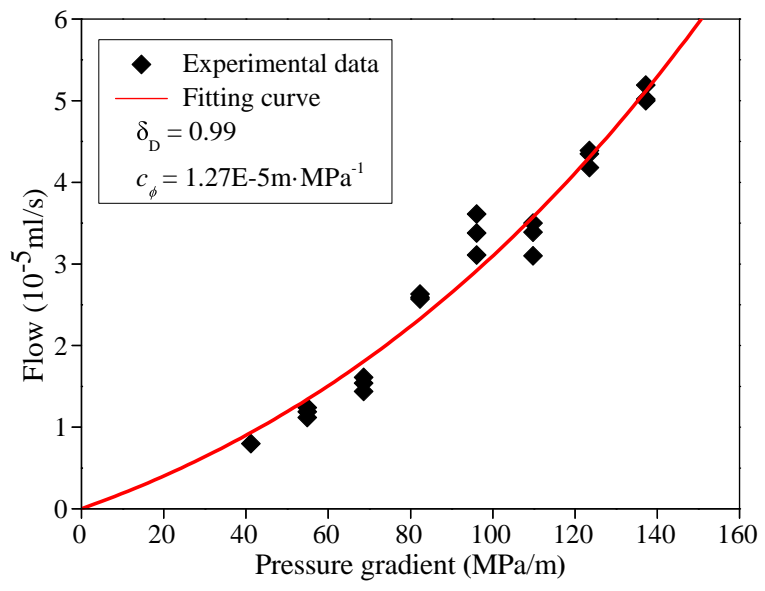

(c)

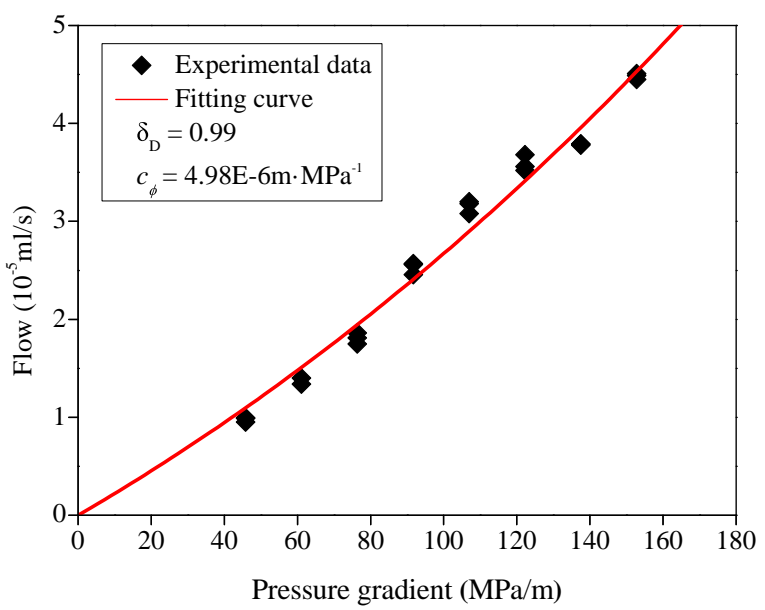

(e)

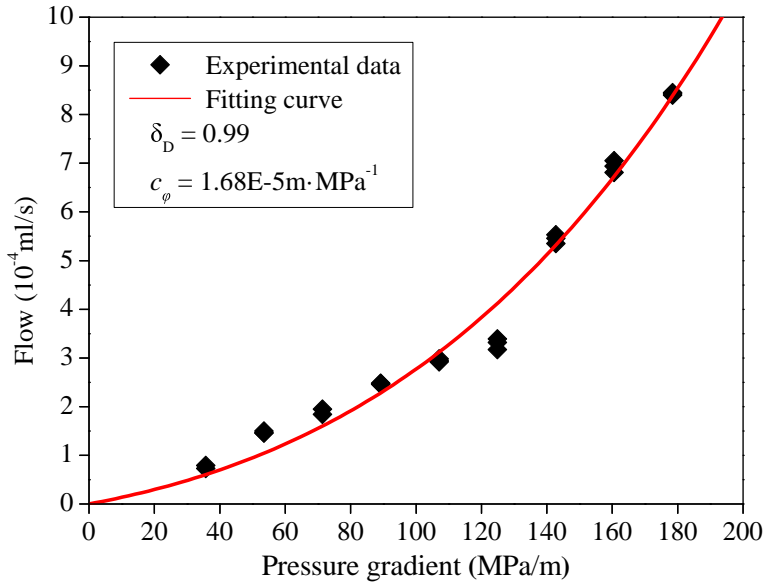

(b)

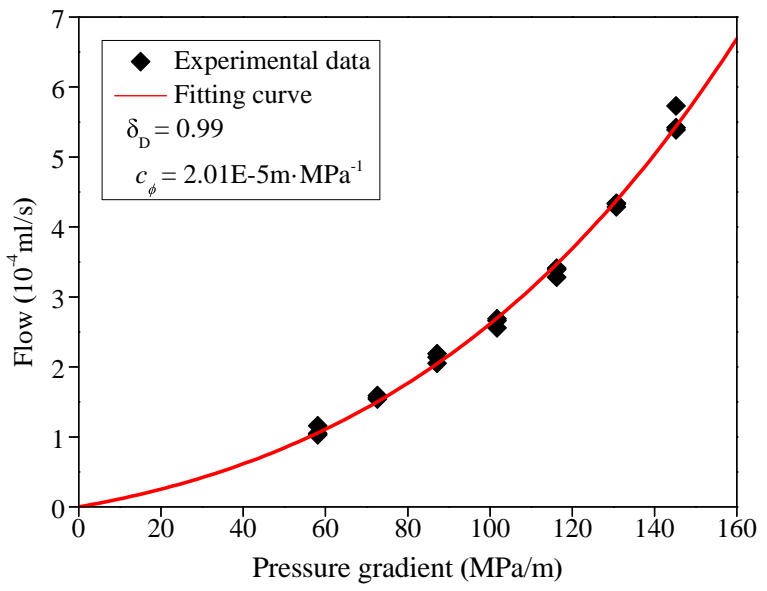

(d)

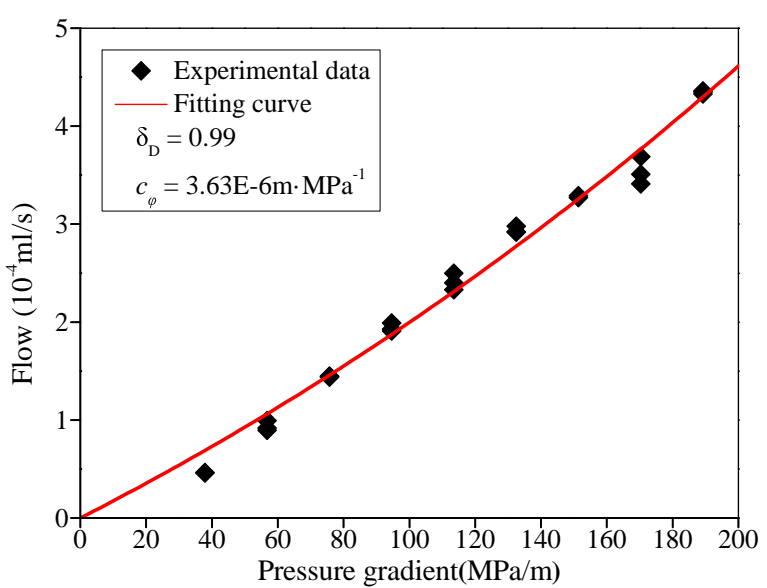

(f)

Fig. 7. The fitting diagram of core nonlinear seepage curve: (a) core-1 $(K=8.33 \mathrm{mD})$; (b) core-2 $(K=7.24 \mathrm{mD})$; (c) core-3 $(K=6.30 \mathrm{mD})$; (d) core-4 (K $=5.87 \mathrm{mD})$; (e) core-5 $(K=5.40 \mathrm{mD})$; and $(\mathrm{f})$ core-6 $(K=4.20 \mathrm{mD})$. 
this research.

Open Access This article is distributed under the terms and conditions of the Creative Commons Attribution (CC BY-NC-ND) license, which permits unrestricted use, distribution, and reproduction in any medium, provided the original work is properly cited.

\section{References}

Deng, Y., Liu, C. Mathematical model of nonlinear flow law in low permeability porous media and its application. Acta Petrolei Sinica 2001, 22(4): 72-77. (in Chinese)

Dong, P., Lei, G. Nonlinear seepage regularity of tight sandstone rese-rvoirs with consideration of medium deformation. Chinese Journal of Rock Mechanics and Engineering 2013, 32(2): 3187-3195. (in Chinese)

Feng, J., Wang, Y., Cui, X., et al. Experimental study on seepage characteristics of low permeability reservoirs. Inn. Mong. Petrochem. Ind. 2010, 36(20): 143-145.

Huang, Y. Non-linear seepage characteristics in low permeability reservoir. Special Oil Gas Reserv. 1997, 4(1): 9-14.

Huang, Y., Yang, Z., He, Y., et al. Nonlinear porous flow in low permeability porous media. Mech. Eng. 2013, 35(5): 1-8.

Jiang, R., Yang, R., Ma, Y., et al. Nonlinear percolation theory and numerical simulation in low permeability reservoirs. Chin. J. Hydrodyn. 2011, 26(4): 444-452.

Li, D., Zha, W., Liu, S., et al. Pressure transient analysis of low permeability reservoir with pseudo threshold pressure gradient. J. Pet. Sci. Eng. 2016, 147: 308-316.

Li, Y., Lei, Q., Liu, X., et al. Characteristic of micro scale nonlinear filtration. Pet. Explor. Dev. 2011, 38(3): 336340.

Li, Z., Zheng, X. The problems and progress in the experimental study of micro/nano-scale flow. J. Exper. Fluid Mech. 2014, 28(3): 1-9.

Liu, X., Liu, X., Hu, Z. On the percolation features of Song $\mathrm{Zi}$ oilfield. Journal of Jianghan Petroleum University of Staff and Workers 2011, 24(3): 6-9. (in Chinese)

Liu, Y. Seepage characteristics and mechanism study of deepburied, high-pressure and low-permeability oil reservoirs in Wendong oilfield. Pet. Geol. Eng. 2015, 29(3): 110-
113.

Peng, C. The study of actuating pressure gradient of the fluid flow in low permeable reservoir. Petroleum Geophysics 2012, 10(1): 64-66. (in Chinese)

Song, F., Song, X., Wang, Y., et al. Single- and two-phase flow model in low-permeability reservoir. Petrolum 2018 (In Press).

Song, F., Tian, H., Zhang, S., et al. The characteristics of deionized water flow in hydrophilic micro-and nano-flow micro-tubes. J. Hydrodyn. 2016, 31(5): 615-619.

Tian, X., Cheng, L., Cao, R., et al. Characteristics of boundary layer in micro and nano throats of tight sandstone oil reservoirs. Chin. J. Comput. Phys. 2016. 33(6): 717-724.

Wang, Q., Tang, H., Lv, D., et al. An experimental study on threshold pressure gradient in low permeability reservoir. Petroleum Geology and Recovery Efficiency 2011, 18(1): 97-100. (in Chinese)

Wei, A., Zhang, L., Zhao, Y. Based on the Origin curve fitting of single phase flow in low permeability reservoirs. Neijiang Science Technology 2011, 32(8): 90-91. (in Chinese)

$\mathrm{Xu}, \mathrm{S}$., Yue, X. Influence of boundary-layer fluid on the seepage cha-racteristic of low-permeability reservoir. Journal of Xi'an Shiyou University (Natural Science Edition) 2007, 22(2): 26-28. (in Chinese)

Yao, Y. Study on non-Darcy flow pattern in low permeability oil reservoir. Xinjiang Petroleum Geology 2000, 21(3): 213-215. (in Chinese)

Zhang, P., Zhang, L., Li, W., et al. Experiment on the influence of boundary layer on the Non-Darcy seepage law. Journal of Hebei University of Engineering (Natural Science Edition) 2008, 25(3): 70-72. (in Chinese)

Zhao, Q., Yue, X., Wang, F., et al. Flow characteristicss of high pressure real gas in microtubes. Journal of China University of Petroleum (Edition of Natural Science) 2012, 36(4): 129-133. (in Chinese)

Zhao, Y., Wang, X., Ling, H., et al. Experimental study on the porous flow law of tight oil reservoir. Science \& Technology Review 2014, 32(28): 59-63. (in Chinese) 